\title{
A Structural Approach to Control of Transitional Waves of Nonlinear Diffusion-reaction Systems: Theory and Possible Applications to Bio-medicine
}

\author{
VICTOR KARDASHOV* and SHMUEL EINAV \\ Department of Biomedical Engineering, Tel-Aviv University, Ramat-Aviv, Tel-Aviv 69978, Israel
}

(Received 10 January 2001; Revised 20 May 2001)

\begin{abstract}
This paper has considered a novel approach to structural recognition and control of nonlinear reactiondiffusion systems (systems with density dependent diffusion). The main consistence of the approach is interactive variation of the nonlinear diffusion and sources structural parameters that allows to implement a qualitative control and recognition of transitional system conditions (transients). The method of inverse solutions construction allows formulating the new analytic conditions of compactness and periodicity of the transients that is also available for nonintegrated systems. On the other hand, using of energy conservations laws, allows transfer to nonlinear dynamics models that gives the possiblity to apply the modern deterministic chaos theory (particularly the Feigenboum's universal constants and scenario of chaotic transitions).
\end{abstract}

Keywords: Density dependent diffusion; Structural interaction; Finite localized transients; Pulse propagations chaos degree

\section{INTRODUCTION: NONLINEAR DIFFUSION- REACTION SYSTEMS AND SELF- ORGANIZATION IN ACTIVE MEDIA}

The self-organization mechanisms in complex nonlinear systems of reaction-diffusion type are considered. In recent paper (Gontar, 2000) the necessity of new principle and mathematical model for the dynamics of complex and living systems is presented. Underlined the need for careful using differential equation (DE) for chaotic and living systems. Solutions of DE and corresponding difference equations should converge when the difference in initial conditions tends to zero. As opposed this, from mathematical point of view, a small amount of information can lead to drastic changes in living systems. On the other hand, in author's paper (Kardashov, 1999) the so called finite control and recognition methods are suggested for investigation of transitional waves (transients) arising in active media with nonlinear diffusion or dispersion features. These methods propose the using of conservation laws and corresponding phase plane portrait for structural analysis of the system transitional conditions, level of selforganization and transition to deterministic chaos. The main component of this approach is interactive variation of nonlinear diffusion and source parameters. A general concept of diffusion stimulation of the evolutionary processes was proposed by Talanov (1983). It should be noted that the nonlinear reaction-diffusion models and corresponding nonlinear phenomena was developed in a lot of recent papers (for example, in (Rosenau and Hyman (1993)) and (Li and Oliver (1997)). Analytical investigations and computer simulation of patterns in blood flow through branches and large vessels are considered by Einav et al. (1990), Elad et al. (1991) and Kardashov and Einav (1997). A constitutive equation for concentrated suspensions that accounts the shear-induced particle migration (Phillips et al., 1992) can be presented as nonlinear reaction-diffusion models of the blood flow in large vessels. On the other hand, in recent papers (see, for example I. Cohen et al., 1998) nonlinear reactiondiffusion models were developed for studies of bacterial branching growth and bacterial cooperative self-organization.

The main goal of this paper is to develop the mathematical models of transitional waves structural self-organization and control associated with nonlinear

*Corresponding author. 
reaction-diffusion systems (see references Sanches-Garduno et al., 1996).

For the most part of recent articles are considered the semi-linear models (starting by the classical work (Kolmogorov et al., 1937)) that describe the processes with linear diffusion and nonlinear sources. Corresponding transients characterized by infinite transition time and unbounded spatial intention. We consider models with nonlinear diffusion that associated with finite localized or periodic transitional waves (FPTW). The nonlinear diffusion-reaction models describe wave propagation in active media (see for instance (Mikhailov, 1991)). Furthermore will be shown the feasibility of these models for mathematical description of pulse propagation in cardio-vascular system, that may be considered as complex self-organization process determined by interaction of blood flow dynamics with input impedance of arteries and vessels (see (Westerhof, 1995)). On the other hand, at recent years the new discrete dynamics of complex and living systems are developing (Gontar and Ilin, 1991; Gontar, 1993; Gontar, 1997). From our point of view, it is possible to implement the common background that is based on applications of the continual conservation laws and discrete methods for investigation of selforganization in active media. Effect of finite localization and periodicity of transients, associated with controlling diffusion or dispersion processes, may be considered as features of self-organization in complex and living active media.

(a) Our approach is based on methods of FPTW recognition and control by interactive variation of the structural parameters of the nonlinear diffusion and nonlinear sources. It is known that the transients corresponding to separatrix lines on the phase plane play a similar role for Hamiltonian diffusion-reaction models to the limit circles in auto-oscillatory systems. Because of existence of the so called separatrix layers, generating chaotic trajectories of Hamiltonian systems (see, for example (Zaslavskiy and Sagdeev, 1991)), transitional waves can be used for recognition of the system transition to deterministic chaos.

On the other hand, as shown an analyze of models with dissipation in biology, chemistry and physics, like models of auto-catalytic reactions, Brusselator model and others, self-organization process are accomplished by its maintenance in closeness of limited circle. Similarly, the self-organization process in Hamiltonian diffusionreaction systems may have been effected by structural management of the transients. Significance of the transients in self-organization of the Hamiltonian systems is determined by its closeness to separatrix layers and possibilities for transition to deterministic chaos that generate infinite sets of variable structures (like fractals and others). It is important that by such approach there is no necessity for construction of an analytic expression for the transients. Sufficiently to obtain from conservation laws formulas determined its important physical characteristics: period, energy etc., consisting the system structural parameters or its ratio. By variation of these structural parameters and using the iterative calculus technique on separatrix line one may estimate the ranges of the transitional process regularity and critical parameters of chaos transitions. On the other hand, the below obtained exact transient solutions can be used for approximation of experimental signals. So, the structural management of the transients allow to control selforganization process and chaotic transitions of dynamic system.

We consider the stationary transients of two different types: finite localized transitional waves (FLTW) and finite switch (or kink) waves (FSW). These transients are of different qualitative structure: FLTW describe local disturbance of the active media and FSW describe a propagation front from one unstable condition to another. By interactive variation of diffusion and sources (reactions) structure may be implemented the qualitative and quantitative control of these transients. It is important that the transformations of the transients one to another may be effected by above-mentioned structural control. These types of control allow us to change a qualitative structure of the process. From our point of view, it may be considered as one of important features of selforganization. For instance, in order for localization (or periodization) of the front propagation in the chemical or biological processes, one can use the structural control of the last type.

(b) An importance of the transients structural investigation follows from below considering energetic approach to estimation of chaos degree by use of calculus of iteration. This approach based on analyze of the transients momentary energy variations by interactive variation of diffusion and reaction structural parameters. Obtained results allow to conclude that chaos degree of the transitional process determined by ratio of some nonlinear characteristics of diffusion and source functions.

It should be noted that considering models describe the conservation law of the real physical values: energy, impulses, mass, etc. Therefore, for the corresponding transition processes the following equalities are fulfilled:

$$
H\left(u, p_{1}, \ldots, p_{m}\right)=C ; \quad\left(p_{i}=\frac{\partial u}{\partial x_{i}}, i=1, \ldots, m\right)
$$

where $H$ is the finite-dimensional function (analogue of the Hamilton's function) or integral relations:

$$
\begin{aligned}
& \int_{G} F\left(u, p_{1}, \ldots, p_{m}\right) \mathrm{d} x=C_{i} \quad(i=1, \ldots, k) \\
& \left(C, C_{i}=\text { const }\right)
\end{aligned}
$$

By using the above-mentioned relations the investigation and control of transients structure may be reduced 
to investigation and parametric control of singular points and levels of the finite-dimensional functions. From the above, the possibility of application of catastrophe theory and discrete nonlinear dynamics to lot of problems in applied mathematics (for biomedical and chemical engineering, prediction of geodynamic, geophysical and ecological processes) is followed. On the other hand, our theoretical results and preliminary computer modeling show the possibility of investigation and control of system chaos degree by the using of the exact analytic expressions for the transients, consisting the important ratio of the structural parameters (see results of the computer experiments).

We consider the processes that may be described by nonlinear non-stationary equation:

$$
\frac{\partial u}{\partial t}=A(u)
$$

where $A$ is nonlinear operator of either of two types:

$$
\begin{gathered}
A_{1}(u)=\Delta \Phi(u)+f(u) \\
A_{2}(u)=\sum_{r=1}^{m} g_{r}\left(\frac{\partial u}{\partial x_{r}}\right) \frac{\partial^{2} u}{\partial x_{r}^{2}}+h(u)
\end{gathered}
$$

Here $\Delta=\sum_{r=1}^{m} \frac{\partial^{2}}{\partial x_{r}^{2}}$ is Laplace operator, $\Phi(s), g_{r}(s)$, $f(s), h(s)$ are real-value functions that determine nonlinear diffusion intensity and nonlinear sources. In present paper we will be investigating the possibility to control of stationary transients only by interactive variation of these structural functions (without output actions). The general equations of this type occur, for instance, in mathematical biology as models of phase transitions in alloys and in porous media with sources, in chemical physics. Similar models were applied to investigation of heat waves propagation in media with nonlinear thermo-conductivity and nonlinear spatial sources. In monograph (Samarskiy et al. (1987)) an effect of the spatial heat localization with unbounded peakings at finite time was considered for wide class of the problems with nonlinear diffusion and sources. On the other hand, we consider furthermore the models that generate periodic or finite localized transients. These equations are suggested as models of dynamic processes with non-linear feed-back relations describing selforganization in some biological and chemical systems, like self-sustaining pulse propagation in cardiovascular system, heat-catalytic chemical reactions, etc. (see references Hashizume, 1988; Diaz, 1985; Demiray, 1997). Systems with finite localized transients were considered in some of recent articles. Above all we shall note (Rosenau and Hyman, 1993; Li and Oliver, 1997) that at first considered the finite localized solutions of soliton type: compactons and peakons. On the other hand, effect of finite localization and periodicity of the stationary transitive waves for general evolutionary equations were considered by one of authors in (Kardashov (1999)). Idea of stimulated diffusion was considered previously in (Talanov (1983)) and in author's paper (Kardashov (1993)), wherein the eigenfunctions of the nonlinear eigenvalue problems for control of strongly nonlinear evolutionary and oscillatory systems were used. In (Biro (1997)) it was shown that the switch waves present an attractor of the solution sets in diffusion-reaction model. In present paper (Part 1) will be considered the problems of the first type.

\section{STRUCTURAL CONTROL AND RECOGNITION OF THE DIFFUSION-REACTION SYSTEMS BY USING THE FINITE SWITCH WAVES (FSW)}

We consider some nonlinear phenomena for processes that may be described by nonlinear reaction-diffusion equation:

$$
\frac{\partial u}{\partial t}=\Delta \Phi(u)+f(u)
$$

where double differentiable nonlinear function $\Phi(u)$ describes the diffusion or dispersion intensity and $f(u)$ is source function, $\Delta$ is Laplace operator.

We look for solutions of traveling-waves type:

$$
u(t, x)=\varphi(s)=\varphi\left(\sum_{r=1}^{m} a_{r} x_{r}+v t\right) ; \quad(v=\text { const })
$$

where numbers $v$ and $a_{r}$ describe velocity and propagation plain of the traveling wave, $\sum_{r=1}^{3} a_{r}^{2}=1$, and focus our attention on the systems with the set of the stationary homogeneous solutions:

$$
\begin{gathered}
\left\{\varphi_{1}, 0, \varphi_{2}\right\} \\
\varphi_{1} \leq 0 \leq \varphi_{2}
\end{gathered}
$$

Here

$$
f(0)=f\left[\varphi_{i}\right]=0 \quad(i=1,2)
$$

The substitution of the expression (2) into Eq. (1) offers following ordinary DE relative to function $\varphi(s)$ :

$$
v \varphi^{\prime}=m[\Phi(\varphi)]^{\prime \prime}+f(\varphi)
$$

Furthermore the sign " $l$ means differentiation with respects to self-similar variable $s=\sum_{r=1}^{m} x_{r}+v t$ and differentiation with respect the variable $\varphi$ (by the convenience of the situation). 


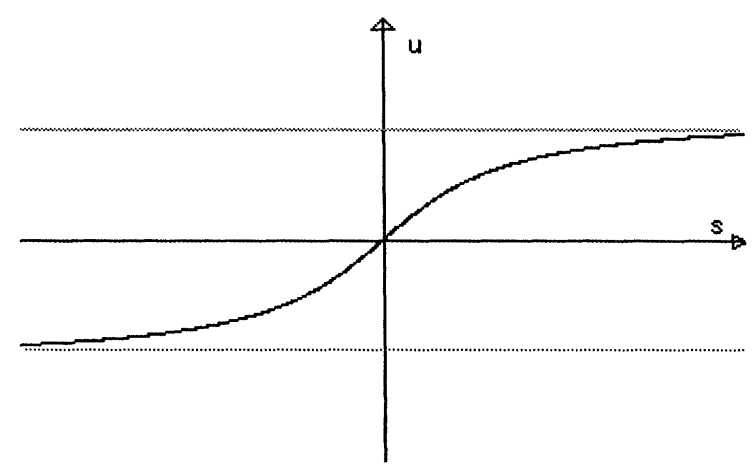

FIGURE 1 Typical switch wave.

Introduce functions:

$$
F(u)=\int_{0}^{u} f(z) \mathrm{d} z ; \quad \Psi(u)=\int_{0}^{u} f(z) \Phi^{\prime}(z) \mathrm{d} z
$$

Definition 1. Bounded solution $\bar{\varphi}(s, v)$ of the Eq. (3) is defined as switch wave (or kink) (SW) if by some numbers $s_{1}, s_{2}$ conditions are fulfilled:

(a) $\lim _{s \rightarrow s_{1}} \bar{\varphi}(s, v)=\varphi_{1} ; \quad \lim _{s \rightarrow s_{2}} \bar{\varphi}(s, v)=\varphi_{2}$;

(b) $\lim _{s \rightarrow s_{1}} \bar{\varphi}^{\prime}(s, v)=\lim _{s \rightarrow s_{2}} \bar{\varphi}^{\prime}(s, v)=0$

(c) $\bar{\varphi}(0, v)=0$

By traditional definitions of the transitional waves $s_{1,2}=$ $\pm \infty$. It is shown below that in problems with nonlinear diffusion exist the transients with finite numbers $s_{1,2}$. In this case the transients will be named finite switch wave (FSW). Typical SW and FSW are presented on Figs. 1 and 2.

(1) In this section we consider the FSW stationarity conditions.

Multiplication of the Eq. (3) by expressions $\frac{\mathrm{d}}{\mathrm{d} s}\{\Phi[\varphi(s)]\}=\Phi_{\varphi}^{\prime}[\varphi(s, v)] \varphi^{\prime}(s, v)$ gives equality:

$$
\begin{aligned}
& v \Phi_{\varphi}^{\prime}[\varphi(s, \nu)]\left[\varphi^{\prime}(s, \nu)\right]^{2} \\
& \quad=\frac{\mathrm{d}}{\mathrm{d} s}\left\{\frac{m}{2}\left[\Phi_{\varphi}^{\prime} \varphi(s, \nu)\right]^{2}\left[\varphi^{\prime}(s, \nu)\right]^{2}+\Psi[\varphi(s, \nu)]\right\}
\end{aligned}
$$

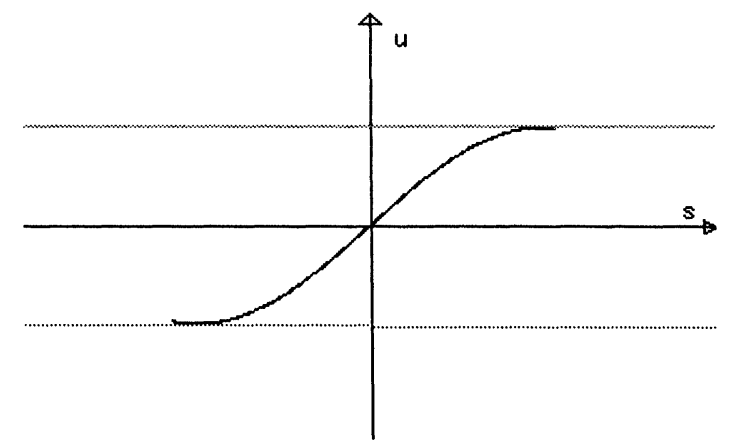

FIGURE 2 Finite switch wave.
It is evident that last equation admits also the supplementary set of stationary solutions governed by finite equation:

$$
\Phi_{\varphi}^{\prime}(\varphi)=0
$$

If the functions $\Phi_{\varphi}^{\prime}, \Psi$ are continuous then the last equality may be rewritten as following:

$$
\begin{aligned}
v \int_{s_{1}}^{s_{2}} \Phi_{\varphi}^{\prime}[\varphi(s, v)]\left[\varphi^{\prime}(s, v)\right]^{2} \mathrm{~d} s \\
=\frac{m}{2}\left\{\Phi_{\varphi}^{\prime}\left[\varphi\left(s_{2}, v\right)\right] \varphi^{\prime}\left(s_{2}, v\right)\right\}^{2} \\
\quad-\frac{m}{2}\left\{\Phi_{\varphi}^{\prime}\left[\varphi\left(s_{1}, v\right)\right] \varphi^{\prime}\left\{\left(s_{1}, v\right)\right\}^{2}+\Psi\left[\varphi\left(s_{2}, v\right)\right]\right. \\
\quad-\Psi\left[\varphi\left(s_{1}, v\right)\right]
\end{aligned}
$$

It will be supposed furthermore that the following condition is fulfilled:

$$
\int_{s_{1}}^{s_{2}} \Phi_{\varphi}^{\prime}[\varphi(s, v)]\left[\varphi^{\prime}(s, \nu)\right]^{2} \mathrm{~d} s \neq 0
$$

Then for the switch waves $\varphi=\bar{\varphi}(s, v, \alpha)$ (see definition 1) last equations imply the important formula:

$$
v=\frac{\Psi\left(\varphi_{2}\right)-\Psi\left(\varphi_{1}\right)}{\int_{s_{1}}^{s_{2}} \Phi_{\varphi}^{\prime}[\bar{\varphi}(s, v)]\left[\bar{\varphi}^{\prime}(s, v)\right]^{2} \mathrm{~d} s}
$$

1. Using the formulas (5) and (6) one may formulate the stationarity conditions for the switch waves.

Theorem 1. Suppose that following conditions are fulfilled:

(a) function $\Phi_{\varphi}^{\prime}(u)$ is bounded at the points $u_{1}=\varphi_{1}$, $u_{2}=\varphi_{2}$

(b) function $\Psi(u)$ is continuous at points $u_{1}, u_{2}$ and $\Psi\left(u_{1}\right)=\Psi\left(u_{2}\right)$

(c) the condition (5) is fulfilled

Then the switch waves are stationary ones.

Proof. From equalities (5) and (6) and conditions of the theorem follows equality $v=0$ that means the stationarity of the switch waves.

It is possible to formulate the other stationarity conditions of the switch waves in processes, described by the Eq. (1).

Suppose that $\Phi(u), f(u)$ are odd functions on variable $u$ and function $\Phi_{\varphi}^{\prime}(u)$ is continuous at points $u_{1}=\varphi_{1}$ and $u_{2}=\varphi_{2}$. 
Integrating the Eq. (3) over interval $\left[s_{1}, s_{2}\right]$ gives:

$$
\begin{gathered}
\bar{v}\left[\bar{\varphi}\left(s_{2}, v\right)-\bar{\varphi}\left(s_{1}, v\right)\right]=\Phi_{\varphi}^{\prime}\left[\bar{\varphi}\left(s_{2}, v\right)\right] \bar{\varphi}^{\prime}\left(s_{2}, v\right) \\
-\Phi_{\varphi}^{\prime}\left[\bar{\varphi}\left(s_{1}, v\right)\right] \bar{\varphi}^{\prime}\left(s_{1}, v\right)+\int_{s_{1}}^{s_{2}} f[\bar{\varphi}(s, v)] \mathrm{d} s
\end{gathered}
$$

It may be shown that on these conditions switch wave $\bar{\varphi}(s)$ is odd function, $s_{2}=-s_{1}, \varphi_{2}=-\varphi_{1}>0$ and integral in last equalities turns to zero. Taking this into account one obtains from last equalities and definition 1:

$$
2 v \varphi_{2}=0
$$

that implies equality $v_{-}=0$.

Furthermore we consider following particular cases:

(a) $f_{3}(u)=a u+b u^{3}, \Phi_{3}(u)=c u+d u^{3}$

(b) $f_{2}(u)=a u+b u^{2}, \Phi_{2}(u)=c u+d u^{2}$

Since $f_{3}(u), \Phi_{3}(u)$ are odd functions, above-mentioned implies that corresponding SW are stationary ones. Using results of theorem 1 one may formulate the conditions of the SW stationarity also in the case (b). Since in this case

$$
\varphi_{0}=0, \varphi_{1}=\varphi_{2}=-\frac{a}{b}
$$

one may obtain the relationship:

$$
\begin{aligned}
\Psi\left(u_{0}\right)-\Psi\left(u_{1}\right) & =\int_{0}^{-a / b}\left(a u+b u^{2}\right)(c+2 d u) \mathrm{d} u \\
& =\frac{a^{3}}{6 b^{2}}\left(c-\frac{a d}{b}\right)
\end{aligned}
$$

Thus, if $a \neq 0$ the condition of the SW stationarity is the following parametric relationship:

$$
\frac{a}{b}=\frac{c}{d}
$$

If $a=0, b \neq 0$ the $S W$ are stationary on arbitrary coefficients $c, d$.

On the other hand, if one will consider the nonstationary transients (on condition $\nu=$ const $\neq 0$ ) then from formula (6) follows the equality:

$$
\begin{aligned}
\int_{s_{1}}^{S} \Phi_{\varphi}^{\prime}[\bar{\varphi}(s, v)]\left[\bar{\varphi}^{\prime}(s, v)\right]^{2} \mathrm{~d} s & =\frac{\Psi[\varphi(S)]-\Psi\left(\varphi_{1}\right)}{v} \\
& =\frac{1}{v} \int_{\varphi_{1}}^{\varphi} f(u) \Phi_{\varphi}(u) \mathrm{d} u
\end{aligned}
$$

on arbitrary $s_{1} \leq S \leq s_{2}, \varphi_{1} \leq \varphi \leq \varphi_{2}$.

The left hand integral may be consider as current value of average kinetic energy $E(S)$ of the transient on given nonlinear diffusion law. The right hand expression presents the one-dimensional function $F(\varphi, v, A)$. Thus, the last equality allow us to reduce the investigation of the nonstationary current energy evolution to structural investigation of the one-dimensional function. Hereby, $A$ is signed the vector of parameters that presents the main diffusion-reaction structural relationships. Application of the discrete dynamics and deterministic chaos methods allow to determine the critical values of the parameters $v$, $A$ on that the bifurcations and chaos transitions are observed. Furthermore it will be used as basis for developing of the discrete energetic methods of chaotic behavior investigation of the nonstationary transients of the considering systems. However, for suitable discretization of the stationary transitional processes furthermore will be used method of inverse solution construction that allows to implement the structural investigation of the transients and also construct the corresponding Poincare map. It is important that such approach is applicable to wide classes (also non-integrable) nonlinear reaction-diffusion systems.

2. Construction of inverse solutions and interactive structural control of the stationary switch waves (SW).

From conditions, that were considered above, follows that stationary SW are governed by ordinary DE:

$$
m[\Phi(\varphi)]^{\prime \prime}+f(\varphi)=0
$$

that obtained from Eq. (3) on $v=0$. By multiplication of these equation by expressions $[\Phi(\varphi)]^{\prime}=\Phi_{\varphi}^{\prime}[\varphi(s)] \varphi^{\prime}(s)$ and consequent integration, one obtains the following sets of the first integrals for this equation:

$$
\frac{m}{2}\left[\Phi_{\varphi}^{\prime}(\varphi)\right]^{2}\left[\varphi^{\prime}\right]^{2}+\Psi(\varphi)=C
$$

The last equality presents the stationary conservation law of the considering process. According to definition of the switch wave

$$
\varphi\left(s_{1}\right)=\varphi_{1} ; \varphi\left(s_{2}\right)=\varphi_{2} ; \varphi(0)=0 ; \varphi^{\prime}\left(s_{1}\right)=\varphi^{\prime}\left(s_{2}\right)=0
$$

Using these equalities and Eq. (8) on values $s=s_{1}$ and $s=s_{2}$ one may determine the arbitrary constant $C$ :

$$
C=\Psi\left(\varphi_{1}\right)=\Psi\left(\varphi_{2}\right)=\Psi_{0}
$$

Particularly, from last formulas follows that stationarity condition (b), considering in above subsection, are both necessary and sufficient.

By separation of the variables in the Eq. (8) one may obtain:

$$
\begin{aligned}
& \frac{\mathrm{d} \varphi}{\mathrm{d} s}= \pm \frac{\sqrt{\frac{2}{m}\left[\Psi_{0}-\Psi(\varphi)\right]}}{|\Phi(\varphi)|} \text { or } \\
& \mathrm{d} s= \pm \frac{|\Phi(\varphi)| \mathrm{d} \varphi}{\sqrt{\left.\frac{2}{m} \mid \Psi_{0}-\Psi(\varphi)\right]}}
\end{aligned}
$$


By integrating the left side of the last equation (considering at first with sign + ) over interval $\left[s_{1}, s\right]\left(s_{1} \leq\right.$ $\left.s \leq s_{2}\right)$ and the right side-over interval $\left[\varphi_{1}, \varphi\right]\left(\varphi_{1} \leq \varphi \leq\right.$ $\varphi_{2}$ ) (taking into account conditions (a),(b)) one may obtain the formula for inverse solution:

$s^{+}(\varphi)=s_{1}^{+}+\int_{\varphi_{1}}^{\varphi} \frac{\left|\Phi^{\prime}(z)\right| \mathrm{d} z}{\sqrt{(2 / m)\left[\Psi_{0}-\Psi(\varphi)\right]}}\left(\varphi_{1} \leq \varphi \leq \varphi_{2}\right)$

Taking into account equalities

$$
\varphi(0)=0, \quad \varphi\left(s_{2}\right)=\varphi_{2}
$$

one may obtain from formula (10):

$$
\begin{aligned}
s_{1}^{+} & =-\int_{\varphi_{1}}^{0} \frac{|\Phi(z)| \mathrm{d} z}{\sqrt{(2 / m)\left[\Psi_{0}-\Psi(z)\right]}} ; \\
s_{2}^{+} & =s_{1}^{+}+\int_{\varphi_{1}}^{\varphi_{2}} \frac{|\Phi(z)| \mathrm{d} z}{\sqrt{(2 / m)\left[\Psi_{0}-\Psi(z)\right]}} \\
& =\int_{0}^{\varphi_{2}} \frac{|\Phi(z)|}{\sqrt{(2 / m)\left[\Psi_{0}-\Psi(z)\right]}}
\end{aligned}
$$

It is evident that last formulas determine monotonically increasing switch wave. Similarly, monotonically decreasing switch wave $\varphi^{-}(s)$ are determined by formula (9), corresponding to sign -

Obtained formulas allow us to investigate the transients structure without construction of its exact analytic expressions. It is especially important for structural investigation of nonintegrated transitional solutions.

Now we consider the conditions of finiteness (compactness) of the switch waves. It is evident that necessary and sufficient condition for transient compactness is finiteness of the numbers $s_{1}$ and $s_{2}$.

By the Taylor's formula one may obtain:

$$
\begin{aligned}
& \Psi_{0}-\Psi(z)=\Psi\left(\varphi_{i}\right)-\Psi(z)=\Psi^{\prime}\left(\varphi_{i}\right)\left(\varphi_{i}-z\right) \\
& -\frac{1}{2} \Psi^{\prime \prime}\left[\varphi_{i}+\theta_{i}\left(z-\varphi_{i}\right)\right]\left(z-\varphi_{i}\right)^{2} \\
& \left(0 \leq \theta_{i} \leq 1 ; i=1,2\right)
\end{aligned}
$$

Since $\Psi^{\prime}\left(\varphi_{i}\right)=f\left(\varphi_{i}\right) \Phi^{\prime}\left(\varphi_{i}\right)=0$ one may obtain from formula (11):

$\left|s_{i}\right|=\int_{0}^{\varphi_{i}} \frac{\left|\Phi^{\prime}(z)\right| \mathrm{d} z}{\sqrt{-(1 / m) \Psi^{\prime \prime}\left\{\varphi_{i}+\theta_{i}\left[z-\varphi_{i}\right]\right\}\left|z-\varphi_{i}\right|}}(i=1,2)$

Taking into account the formulas (10), (11) and (12) one may formulate a following necessary and sufficient finiteness conditions for the switch waves in problem (1):

$$
\begin{aligned}
& \text { (a) }\left|\Phi^{\prime}(z)\right|=\mathrm{O}\left(|z|^{\gamma_{0}}\right) \quad \text { on } \quad z \rightarrow 0, \quad \gamma_{0}>-1 \text {; } \\
& \text { (b) }\left|\Phi^{\prime}(z)\right|=\mathrm{O}\left(\left|z-\varphi_{1}\right|^{\gamma_{1}}\right) \quad \text { on } \quad z \rightarrow \varphi_{1} \text {, } \\
& \gamma_{1}>0 ; \quad(\mathrm{c})\left|\Phi^{\prime}(z)\right|=\mathrm{O}\left(\left|z-\varphi_{2}\right|^{\gamma_{2}}\right) \quad \text { on } \\
& z \rightarrow \varphi_{2}, \quad \gamma_{2}>0 ; \quad \text { (d) function } \quad \Psi^{\prime \prime}(z) \leq 0
\end{aligned}
$$

on $\left[\varphi_{1}, \varphi_{2}\right]$ and continuous in points

$\varphi_{1}, \varphi_{2}$

Since

$$
\Psi^{\prime \prime}(z)=f^{\prime}(z) \Phi^{\prime}(z)+f(z) \Phi^{\prime \prime}(z)
$$

and $\varphi_{\mathrm{i}}$ are simple roots of the function $f(u)$ then

$$
\Psi^{\prime \prime}(z)=O\left[\left|\Phi^{\prime}(z)\right|\right] \quad \text { on } \quad \varphi \rightarrow \varphi_{i}(i=1,2)
$$

Taking into account last relationship and conditions $(13(a-d))$, one may obtain that

$$
\begin{aligned}
\Phi_{1}(z) & =\frac{\left|\Phi^{\prime}(z)\right|}{\sqrt{-(2 / m) \Psi^{\prime \prime}\left\{\varphi_{i}+\theta_{i}\left[z-\varphi_{i}\right]\right\}\left|z-\varphi_{i}\right|}} \\
& =O\left(\mid z-\varphi_{i}\right) \mid\left(\gamma_{i} / 2^{-1}\right)\left(\gamma_{i}>0\right)
\end{aligned}
$$

and

$$
\Phi_{1}(z)=\mathrm{O}\left(|z|^{\gamma_{0}}\right)\left(\gamma_{0}>-1\right) \quad \text { on } \quad z \rightarrow 0
$$

From last relationships and formulas (9)-(12) follows the finiteness of switch waves.

Above-mentioned conditions (13) can be satisfied by use of following simple procedure that may be characterized as method of diffusion and reaction singular points coincidence.

Furthermore, we consider a particular case:

$$
f(u)=a u+b u^{3}, \quad \Phi(u)=c u+d u^{3}
$$

Since $f(u), \Phi(u)$ are odd functions, according abovementioned the switch waves present the stationary transients.

It will be shown that for this case on conditions (13) the FSW are periodic waves determined by simple harmonical function. It is evident that nontrivial roots of the functions $f(u)$ and $\Phi^{\prime}(u)$ are determined by formulas:

$$
\varphi_{1,2}= \pm \sqrt{-\frac{a}{b}}, \psi_{1,2}= \pm \sqrt{-\frac{c}{3 d}}
$$

It is evident that conditions (13) follow from relationship:

$$
\frac{a}{b}=\frac{c}{3 d}
$$




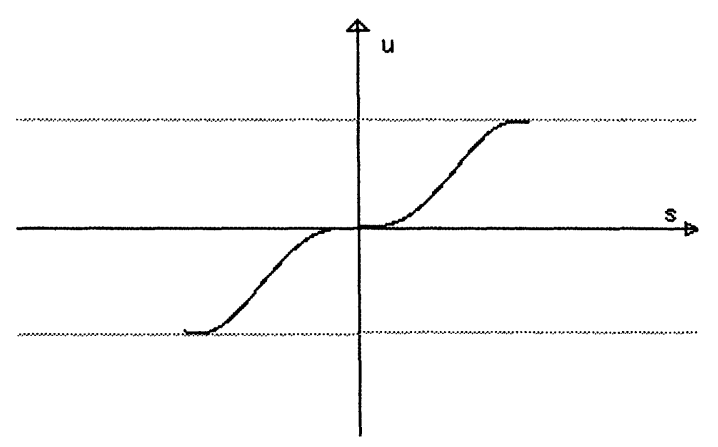

FIGURE 3 FSW with infinite small velocity at zero.

Taking this into account one may obtain that

$$
\begin{aligned}
\Psi^{\prime} & =f(u) \Phi^{\prime}(u)=\left(a u+b u^{3}\right)\left(c+3 d u^{2}\right) \\
& =\frac{b}{3 d} u\left(u^{2}+\frac{a}{b}\right)^{2}
\end{aligned}
$$

By integration of last expression one may obtain the formula:

$$
\Psi(u)=\frac{b}{18 d}\left(u^{2}+\frac{a}{b}\right)^{3}
$$

thus

$$
\Psi_{0}=\Psi\left( \pm \sqrt{-\frac{a}{b}}\right)=0
$$

On the other hand, it is evident from above-mentioned formulas that

$$
\Phi^{\prime}(u)=c+3 d u^{2}=\frac{1}{3 d}\left(\frac{a}{b}+u^{2}\right)
$$

The general formula (10) together with last relationship imply the following expression for inverse function to solution:

$$
\begin{aligned}
s(\varphi) & =s_{1}+\sqrt{2\left|\frac{b}{d}\right|} \int_{-\sqrt{\left|\frac{|a|}{b}\right|}}^{\varphi} \frac{\mathrm{d} u}{\sqrt{\left|\frac{a}{b}\right|-u^{2}}} \\
& =s_{1}+\sqrt{2\left|\frac{b}{d}\right|}\left[\arcsin \left(\sqrt{\left|\frac{b}{a}\right|}\right) \varphi+\frac{\pi}{2}\right]
\end{aligned}
$$

The constants $s_{1,2}$ may be determined according to formula (11):

$$
\begin{aligned}
& s_{1}=-\sqrt{2\left|\frac{b}{d}\right|} \int_{0}^{\sqrt{\left|\frac{a}{b}\right|}} \frac{\mathrm{d} u}{\sqrt{\left|\frac{a}{b}\right|-u^{2}}}=-\sqrt{2\left|\frac{b}{d}\right| \frac{\pi}{2}} ; \\
& s_{2}=-s_{1}=\sqrt{2\left|\frac{b}{d}\right| \frac{\pi}{2}}
\end{aligned}
$$

Taking into account two last formulas one may obtain the exact expressions for pair of two FSWs:

$$
\varphi_{1,2}(s)= \pm \sqrt{\left|\frac{b}{a}\right| \sin }\left(\sqrt{2\left|\frac{b}{d}\right| s}\right)
$$

Now we shall do the important comment.

If for the problem (1) is accomplished the condition (a) alone, the switch waves asymptotically (on indefinite time) approaches to values $\varphi_{1}, \varphi_{2}$ (see Fig. 1.) It immediately implies an important conclusion: finiteness, periodicity and structural stability of the FSW is accomplished on special growth limitation of the diffusion function $\Phi^{\prime}(u)$ in closeness of the zeroth points of source functions $f(u)$. By this interaction the feedback control of the switch waves structure can be implemented. The functions $\varphi_{1,2}(s)$ determine the pair of FSW that may be periodically continued (see last formulas).

It is evident that by variation of the parameters $\gamma_{0}, \gamma_{1}, \gamma_{2}$ one may change some important geometric characteristics of the FSW. Formula (9) implies that on condition $-1<$ $\gamma_{0}<0$ one obtains FSW, tangent to axis $\mathrm{O}_{x}$ at the point $x=0$ (see Fig. 3). Physically this means that velocity of the transitional process at zero is infinite small value of the order $\gamma_{0}$. On $\gamma_{0}>1$ the FSW is depicted by curve that tangent to axis $\mathrm{O}_{y}$ at the origin (see Fig. 4). Physically this means that velocity of transitional process has infinite growing value of the order $\gamma_{0}$.

Then, by choosing the diffusion law of type

$$
\Phi(u)=A u^{\gamma_{0}}\left(u-\varphi_{1}\right)^{\gamma_{1}}\left(u-\varphi_{2}\right)^{\gamma_{2}}
$$

and by corresponding management of the parameters $\gamma_{0}$, $\gamma_{1}, \gamma_{2}$ one may implement above-mentioned feedback structural control of the transitional process. On the other hand, using these results, one may formulate the following approach to optimal control of diffusion-reaction process by interaction of the diffusion and reaction parameters, using obtained formulas for FSW.

For instance, the important problem for applications is construction of the transitional processes with minimal transient time $T=s_{2}-s_{1}$. For periodic FSW the magnitude $T=s_{2}-s_{1}$ determines period or frequency of the transient and, consequently, its kinetic energy. On the other hand, it may be shown that the value of period, frequency and kinetic energy of the FSW depict the extremal ones for definite set of the periodic solutions of the problem. Thus by considering structural interaction of the FSW parameters one may implement, in determine sense, the global optimal control of the process.

It is evident that the values $\varphi_{1}, \varphi_{2}$ are functions of structural coefficients of the source function. For instance, if $f(u)=a u+b u^{3}$ then

$$
\varphi= \pm \sqrt{-\frac{a}{b}}= \pm \lambda
$$

Thus, transient time Tis a function of the four structural 


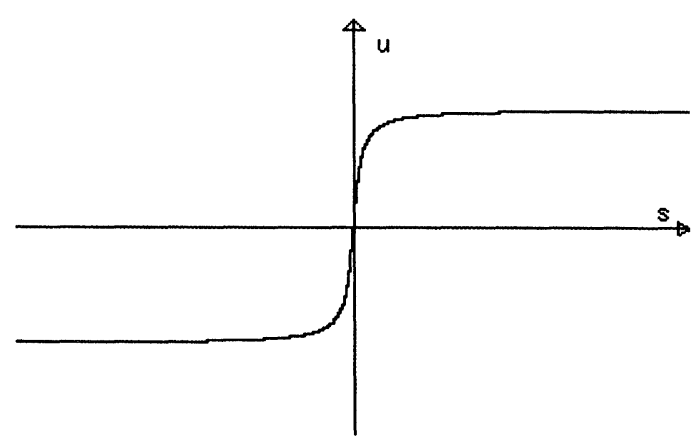

FIGURE 4 FSW with infinite velocity at zero.

parameters:

$$
T=T\left(\gamma_{0}, \gamma_{1}, \gamma_{2}, \lambda\right) \text {. }
$$

One may consider the optimization problems by this finite-dimensional function on corresponding set of the vectors $A\left(\gamma_{0}, \gamma_{1}, \gamma_{2}, \lambda\right)$, according to some criteria. The simple and commonly accepted criteria is minimization of the root-mean square deflection from given constant condition, i.e. of the value:

$$
\mathrm{d}\left(\gamma_{0}, \gamma_{1}, \gamma_{2}, \lambda\right)=\max _{A \in M}\left|T_{0}-T\left(\gamma_{0}, \gamma_{1}, \gamma_{2}, \lambda\right)\right|^{2}
$$

In common case this is a problem of finite-dimensional mathematical programming theory. A set of the solutions are vector $A_{0}$ of the structural parameters, that determine the most interactable reaction-diffusion systems.

Applications is also important for the problem of optimal structural variability of reaction-diffusion system, that is given by criteria:

$$
\rho\left(S_{0}, S[A]\right) \leq \varepsilon
$$

here $S_{0}$ is desirable condition of the system, $S[A]$-set of the allowable structural variability conditions, $\varepsilon>0$ is given number, that determines allowable (or necessary) variability of the system, $\rho(X, Y)$ is metric function, that determines the closeness of the system conditions. To this problem the mathematical programming theory can also be applied.

On the other hand, one may consider the inverse problem, i.e. the problem of dynamic identification of the system by using the experimental and visualization data. The problem is to determine (or estimate) the unknown transitional condition $S_{0}$ of the system by using of the known parameters of conditions $S[A]$, that is obtained by experiments with exactness $\varepsilon$. Here the methods of nonlinear filtration and experimental signals processing can be applied.

\section{STRUCTURAL CONTROL OF THE FINITE LOCALIZED TRANSITIVE WAVES (FLTW). PERIODICITY PHENOMENA FOR FLTW ON DIFFUSION AND REACTION STRUCTURAL INTERACTION}

Now we will consider a concepts of finite localized transient wave (FLTW).
Definition 2. A solution $\varphi(s, v)$ of Eq. (4) is localized transient wave (LTW) if by some numbers $s_{1}, s_{2}$ assert conditions:

(a) $\lim _{s \rightarrow s_{1}} \varphi(s, v)=\lim _{s \rightarrow s_{2}} \varphi(s, v)=0$

(b) $\lim _{s \rightarrow s_{1}} \varphi^{\prime}(s, v)=\lim _{s \rightarrow s_{2}} \varphi^{\prime}(s, v)=0$

(c) $\varphi(0, v)=\varphi_{0} \quad$ or $\varphi(0, v)=\bar{\varphi}_{0}$

where $\varphi_{0}>0$ and $\bar{\varphi}_{0}<0$ are the roots of the equation:

$$
\Psi(\varphi)=0
$$

The typical LTW is presented on Fig. 5.

From definition 2 follow that LTW consistent with unstable critical point $(0,0)$ of the functions $\Psi(u)$.

1. Conditions of stationarity of the localized transitive wave may be obtained similarly by using equality (6) and taking into account the definition 2 of the LTW.

Theorem 2. Suppose that the following conditions are fulfilled

(a) the function $\Phi_{\varphi}^{\prime}(u)$ continuous in zero, the function $\Psi(u)$ is nonpositive on the sets $\left[\varphi_{0}, 0\right],\left[0, \varphi_{0}\right]$. Here $\bar{\varphi}_{0}, \varphi_{0}$ are negative and positive roots of the function $\Psi(u)$;

(b) the following conditions are fulfilled: $\Psi_{u}^{\prime}(0)=$ $f(0)=0$

(c) the condition (5) is fulfilled. On these conditions LTW is stationary wave and governed by Eq. (7).

2. Taking into account the last equalities and substituting into Eq. (8) values $\varphi=0, \varphi^{\prime}=0$, one may obtain that $C=0$.

Furthermore, from equality

$$
\frac{m}{2}\left[\Phi_{\varphi}^{\prime}(\varphi)\right]^{2}\left[\varphi^{\prime}\right]^{2}+\Psi(\varphi)=0
$$

one may obtain the following equations:

$$
\frac{\mathrm{d} \varphi}{\mathrm{d} s}= \pm \underline{\sqrt{-(2 / m) \Psi(\varphi)}\left|\Phi^{\prime}(\varphi)\right|}
$$

By using variables separation method the formulas for inverse functions to LTW of positive polarity can be obtained on intervals $\left[s_{1}, 0\right],\left[0, s_{2}\right]$ respectively:

$$
\begin{array}{r}
s_{1}^{+}(\varphi)=s_{1}^{+}+\int_{0}^{\varphi} \frac{\left|\Phi_{z}^{\prime}(z)\right|}{\sqrt{(-2 / m) \Psi(z)}} \mathrm{d} z \\
s_{1}^{+}=-\int_{0}^{\varphi_{0}} \frac{\left|\Phi_{z}^{\prime}(z)\right|}{\sqrt{(-2 / m) \Psi(z)}} \mathrm{d} z,\left(0 \leq \varphi \leq \varphi_{0}\right) \\
s_{2}(\varphi)=s_{2}^{+}-\int_{\varphi_{0}}^{\varphi} \frac{\left|\Phi^{\prime}(z)\right|}{\sqrt{(-2 / m) \Psi(z)}} \\
s_{2}^{+}=-s_{1}^{+}=\int_{0}^{\varphi_{0}} \frac{\left|\Phi^{\prime}(z)\right|}{\sqrt{(-2 / m) \Psi(z)}}
\end{array}
$$




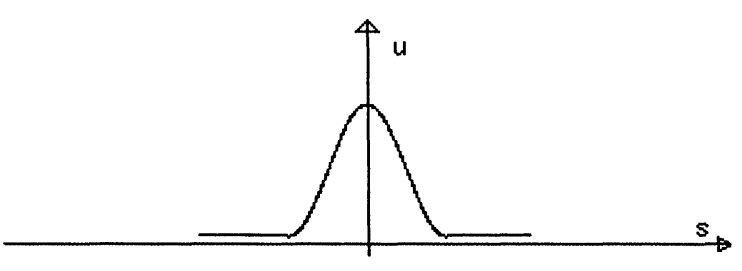

FIGURE 5 Typical localized transient wave.

In a similar way formulas for LTW of negative polarity may be obtained

$$
\begin{aligned}
s_{2}^{-}(\varphi, \alpha) & =\int_{\varphi_{\theta}-}^{\varphi} \frac{\left|\Phi^{\prime}(z)\right|}{\sqrt{(-2 / m) \Psi(z)}}, \\
s_{2}^{-} & =\int_{\varphi_{0}-}^{0} \frac{\left|\Phi^{\prime}(z)\right|}{\sqrt{(-2 / m) \Psi(z)}} \mathrm{d} z,\left(\varphi_{\theta} \leq \varphi \leq 0\right) \\
s_{1}^{-}(\varphi) & =s_{1}^{-}-\int_{0}^{\varphi} \frac{\left|\Phi^{\prime}(z)\right|}{\sqrt{(-2 / m) \Psi(z)}} \mathrm{d} z, \\
s_{1}^{-} & =-s_{2}^{-}=\int_{0}^{\varphi_{\sigma}} \frac{\left|\Phi^{\prime}(z)\right|}{\sqrt{(-2 / m) \Psi(z)}} \mathrm{d} z
\end{aligned}
$$

To take necessary and sufficient conditions of the finiteness (compactness) for LTW one should determine the finiteness conditions for the values $s_{1}^{ \pm}$and $s_{2}^{ \pm}$. Suppose that

$$
\begin{aligned}
& \Phi(z)=\mathrm{O}\left(|z|^{k}\right) \\
& f(z)=\mathrm{O}\left(|z|^{l}\right)
\end{aligned}
$$

on $z \rightarrow 0$

Then necessary and sufficient condition of the LTW finiteness is an inequality:

$$
k>l
$$

It follows from the relationship

$$
\frac{\Phi^{\prime}(z)}{\sqrt{-2 \Psi(z)}}=\mathrm{O}\left(|z|^{(k-l-2) / 2}\right)
$$

on $z \rightarrow 0$ that may be obtained from conditions (20) and (21) and definition of the function $\psi(z)$. It is evident from last equality that necessary and sufficient conditions for finiteness of the integrals $s_{1,2}^{ \pm}$determined by inequality

$$
\frac{k-l-2}{2}>-1
$$

that implies the inequality (22). Thus, an inequality (22) presents the condition of finite localized transitive waves (FLTW) formation. On Fig. 6 a typical FLTW is presented.
Furthermore we consider the particular case:

$$
f=a u+b u^{2}, \Phi(u)=c u+d u^{2}
$$

By formula $\Psi(u)=\int_{0}^{u} f(z) \Phi^{\prime}(z) \mathrm{d} z$ one can obtain that for this case

$$
\Psi(u)=\frac{1}{2} a c u^{2}+\frac{1}{3}(2 a d+b c) u^{3}+\frac{1}{2} b d u^{4}
$$

According to finite localization conditions (20)-(22) one shall to assume $c=0$ that implies the equality:

$$
\Psi_{0}(u)=\frac{2}{3} a d u^{3}+\frac{1}{2} b d u^{4}
$$

Furthermore we suppose that

$$
b>0, d>0, a<0
$$

It is evident that the value $\varphi_{0}=0$ is three multiple root of the function $\psi_{0}(u)$.Nontrivial root of the function $\psi(u)$ is determined by formula:

$$
\varphi_{1}=-\frac{4 a}{3 b}
$$

If the conditions $b>0, d>0, a<0$ are fulfilled the root $\varphi_{1}$ is positive and may be used the formula (16):

$$
\begin{aligned}
s(\varphi) & =s_{1}+2 \sqrt{2} \sqrt{\frac{d}{b}} \int_{0}^{\varphi} \frac{\mathrm{d} u}{\sqrt{(-4 a / 3 b) u-u^{2}}} \\
& =s_{1}+2 \sqrt{2} \sqrt{\frac{d}{b}} \arcsin \left[\frac{3 b}{2|a|}\left(\varphi-\frac{2|a|}{3 b}\right)\right]
\end{aligned}
$$

By inversion of the last formula we obtain:

$$
\varphi(s)=\frac{2|a|}{3 b}\left\{1+\sin \left[\frac{1}{2 \sqrt{2}} \sqrt{\frac{d}{b}}\left(s-s_{1}\right)\right]\right\}
$$

Furthermore, since by formula (17)

$$
s_{1}=2 \sqrt{2} \sqrt{\frac{d}{b}} \frac{\pi}{2}
$$

the last expression may be presented like:

$$
\varphi(s)=\frac{4|a|}{3 b} \cos ^{2}\left(\frac{1}{4 \sqrt{2}} \sqrt{\frac{d}{b}} s\right)
$$

The exact formula (23) corresponds to harmonic FLTW of positive polarity.

Similarly, on conditions.

$$
b<0, \quad d<0, \quad a>0
$$




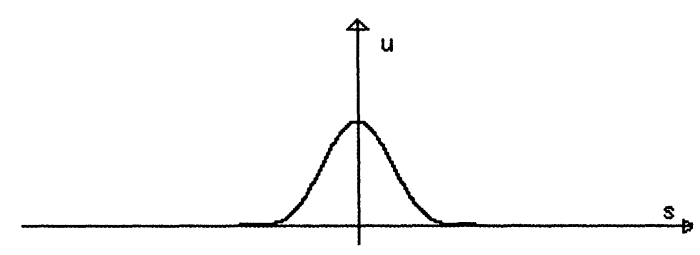

FIGURE 6 Finite localized transient wave.

by using the formulas (18) and (19) one may obtain the expression, corresponding to FLTW of negative polarity:

$$
\varphi(s)=-\frac{4 a}{3|b|} \cos ^{2}\left(\frac{1}{4 \sqrt{2}} \sqrt{\frac{d}{b}} s\right)
$$

Since formula (15) implies the relationship

$$
\frac{\mathrm{d} \varphi}{\mathrm{d} s}=\mathrm{O}\left(|\varphi|^{(2-k+l) / 2}\right) \quad \text { on } \quad z \rightarrow 0
$$

one may conclude that on condition $k+l<2$ is fulfilled:

$$
\frac{\mathrm{d} \varphi}{\mathrm{d} s} \rightarrow 0 \quad \text { on } \quad \varphi \rightarrow 0
$$

Thus, if the system of conditions is fulfilled

$$
k>l, \quad 0<k+l<2
$$

the FLTW are determined by following general formula:

$$
\begin{aligned}
& \varphi^{ \pm}(s)= \pm \frac{4|a|}{3 b} \cos ^{2}\left(\frac{1}{4 \sqrt{2}} \sqrt{\frac{d}{b}} s\right) \text { on condition } \\
& \left|\frac{1}{\sqrt{2}} \sqrt{\frac{d}{b} s}\right| \leq 2 \pi \\
& \varphi^{ \pm}(s)=0 \text { on condition }\left|\frac{1}{\sqrt{2}} \sqrt{\frac{d}{b}} s\right|>2 \pi .
\end{aligned}
$$

In Fig. 6 is presented the graph of the finite localized transient of positive polarity. These exact analytic expressions allow us to investigate and control the system chaos degree by variation of the parameter $L=\sqrt{\frac{d}{4 b}}$ (see on Fig. 7 the bifurcation diagram and on Fig. 8 the momentary energy level oscillations on variations of parameter $L$ ):

This particular case indicates that by sign changing of the structural parameters the transitional process may be switched from positive FLTW to negative one. Obtained basic transients can be used for construction of the transitional processes of more complex structure (for instance for modeling of interaction between forward and backward waves in cardiovascular system and for using them as basis for Fourier expansion, see (Westerhof, (1995)). In the next section we consider a possibility of approximation of experimentally registered solitary pulses in arteries by above-mentioned FLTW.
From formulas (15) follows that important geometric properties of the FLTW and its behavior on $s \rightarrow s_{1,2}$ determined by order of decreasing of $\varphi^{\prime}(s)$ to zero on $\varphi \rightarrow 0$. It was shown from formulas (15), (20) and (21) that on conditions.

$$
0<k-l<2
$$

is fulfilled the relationships:

$$
\varphi^{\prime}(s) \rightarrow 0 \quad \text { on } \quad \varphi \rightarrow 0
$$

This situation may be named as "soft finite localization". On condition

$$
k-l>2
$$

one may obtain from above-mentioned formulas that

$$
\varphi^{\prime} \rightarrow \pm \infty \quad \text { on } \quad \varphi \rightarrow 0
$$

Last situation may be named as "rigid localization" (see Fig. 9).

On condition

$$
k-l=2
$$

FLTW has on $s=s_{1,2}^{ \pm}$finite nonzero derivatives and transfer to periodic switch wave on oddness condition for the functions $\Phi(u)$ and $f(u)$. This is interesting phenomena of the transitional waves structure changing on determine (critical) relationship between growth order of diffusion and source functions.

We consider, for instance, the equation

$$
\frac{\partial u}{\partial t}=\Delta\left(c u^{k+2}\right)+a u^{k}+b u^{m}
$$

with constants $a<0 ; b, c>0$ for odd numbers $k \geq 0$, $m>k$. Using general formulas (16)-(19) one may obtain:

$$
\begin{aligned}
s_{1}^{+}(\varphi) & =s_{1}^{+}+c(k+2) \sqrt{\frac{b}{(2 k+4)}} \int_{0}^{\varphi} \frac{\mathrm{d} u}{\sqrt{A-u^{2}}} \\
& =s_{1}^{+}+c(k+2) \sqrt{\frac{b}{(2 k+4)}} \arcsin \left[\frac{\varphi}{\sqrt{A}}\right]
\end{aligned}
$$

Here $A=(2 k+4)|a| /(2 k+2) b$. Since $s_{1}^{+}(\sqrt{A})=0$ from last formula follows:

$$
s_{1}^{+}=-c(k+2) \int_{0}^{\sqrt{A}} \frac{\mathrm{d} u}{\sqrt{A-u^{2}}}=-c(k+2) \frac{\pi}{2}
$$

Taking into account last two formulas one may obtain the following analytic expression for corresponding 


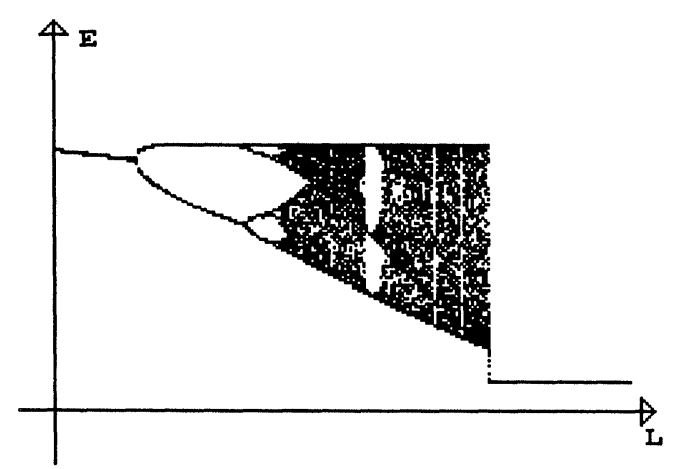

FIGURE 7 Bifurcation diagram of pulse energy evolution.

transitional wave:

$$
\begin{aligned}
\varphi_{k}^{+}(s) & =\sqrt{A_{k}} \sin \left[\frac{1}{k+2} \sqrt{\frac{(2 k+4)}{b}} s+\frac{\pi}{2}\right] \\
& =\sqrt{A_{k}} \cos \left[\frac{1}{c(k+2)} \sqrt{\frac{(2 k+4)}{b}} s\right]
\end{aligned}
$$

Evidently that solution of negative polarity is given by formula:

$$
\varphi_{k}^{-}(s)=-\varphi^{+}(s)
$$

We will consider now two special cases: $k=0,1$. In case $k=0$ one obtains equation with nonlinear diffusion coefficient $\Phi^{\prime}(u)=2 c u$ and reaction function $f(u)=a+b u^{2}$ :

$$
\frac{\partial u}{\partial t}=\sum_{i=1}^{m} \frac{\partial}{\partial x_{i}}\left(2 c u \frac{\partial u}{\partial x_{i}}\right)+a+b u^{2}
$$

As follows from general expression (26) transient solution of this problem are given by the following formulas:

$$
\varphi_{0}^{ \pm}(s)= \pm \sqrt{\frac{2|a|}{b}} \cos \left(\frac{1}{2 \sqrt{b c}} s\right)
$$

in case $k=1$ we obtain an equation:

$$
\frac{\partial u}{\partial t}=\sum_{i=1}^{m} \frac{\partial}{\partial x_{i}}\left(3 c u^{2} \frac{\partial u}{\partial x_{i}}\right)+a u+b u^{3}
$$

with transient solutions:

$$
\varphi_{1}^{ \pm}(s)= \pm \sqrt{\frac{3|a|}{2 b}} \cos \left(\frac{\sqrt{6}}{3} \frac{1}{\sqrt{b} c} s\right)
$$

\section{REDUCTION TO DISCRETE DYNAMICS. ENERGETIC APPROACH TO RECOGNITION AND CONTROL OF CHAOS TRANSITIONS}

The foregoing method of inverse transient solutions construction may be used for transition to discrete dynamic models. Particularly, obtained formula for FLT of the positive polarity

$$
s_{1}^{+}(\varphi)-s_{1}^{+}=\int_{0}^{\varphi} \frac{\left|\Phi_{z}^{\prime}(z)\right|}{\sqrt{(-2 / m) \Psi(z)}} \mathrm{d} z=F(\varphi)
$$

allow for given number of values $s_{n}(n=1, \ldots, N)$ to determine the corresponding recurrent sequence

$$
\varphi_{n+1}=F\left(\varphi_{n}\right)
$$

that is necessary for Poincare map construction. One

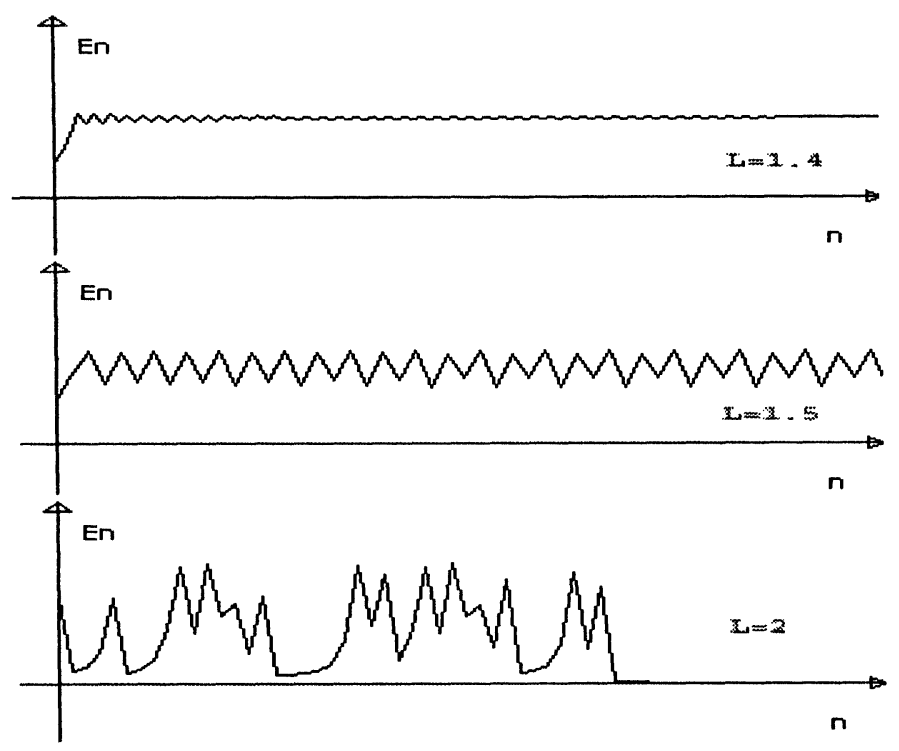

FIGURE 8 Momentary energy levels variation on transition to chaos. 


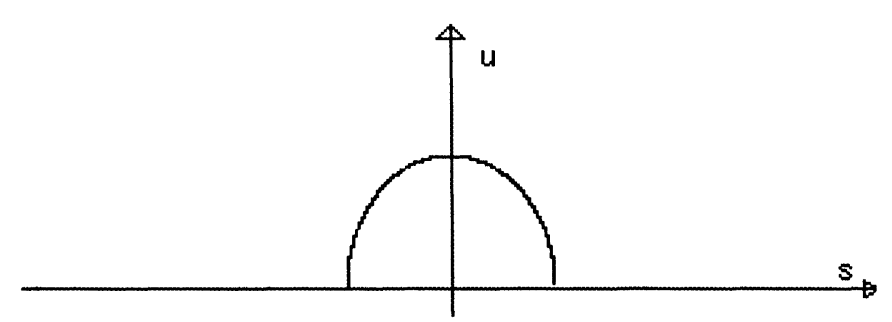

FIGURE 9 Rigidly localized transient.

should find the inverse function (that evidently exist)

$$
F_{1}=F^{-1}
$$

Then the desired recurrent sequence may be presented as like:

$$
\varphi_{n+1}=F_{1}\left[s_{n+1}-s_{n}+F\left(\varphi_{n}\right)\right]
$$

This procedure in oppose using corresponding difference equation has the finite nature and stripped of abovementioned nonstability features.

Now we consider the brief description of the abovementioned approach that use the energy conservation laws for chaos transitions estimation and control. If one introduce the instantaneous total energy

$$
E(s)=\left[\Phi^{\prime}(\varphi)\right]^{2}\left[\varphi^{\prime}(s)\right]^{2}+F[\varphi(s)]
$$

the equality (4) for arbitrary $s \in\left[s_{1}, s_{2}\right]$ may be rewritten as follows:

$$
\int_{s_{1}}^{s} \Phi[\varphi(t)]\left[\varphi^{\prime}(t)\right]^{2} \mathrm{~d} s=\frac{E(s)-E\left(s_{1}\right)}{v}
$$

on condition $v=0$ and as

$$
E(s)=E\left(s_{1}\right)
$$

On condition $v=0$. It is evident that the equality (29) express the important property of the transients: average kinetic energy of the nonstationary transient at arbitrary moment equal to ratio of instantaneous total energy to traveling waves velocity. Equality (30) express a law of total energy conservation for stationary transient. It is proposed to use this energy relationship for estimation of the transient's structural stability and critical relationships between determined system parameters on that the process transfers to deterministic chaos. The expression in the left side of equality (29) determines an average kinetic energy of the transient at arbitrary moment $s_{1} \leq s \leq s_{1}$. On the other hand, if one assume $v=$ const, then the expression in right side determines the ratio of current potential energy to waves velocity. Thus, such approaches allow us to reduce the stability estimation problem of the complex dynamic system (generally non-integrated) for the investigation of two-dimensional function stability and transitions to chaos by use of corresponding iterative sequences technique. Generally these are the catastrophe theory problems (see, for example, (Gilmore, 1981)). It may be also used the recent methods of nonlinear discrete dynamics and iteration calculus. On the other hand, by using of the above-mentioned exact analytic solutions, depending distinctly from structural parameters, can be determined the exact critical constants, on that the system transfer to deterministic chaos. Our computer experiments (see Figs. 7 and 8) allow to formulate two important preliminary conclusions:

(a) bounds of the system stability and system chaos degree depend on the relationship of diffusion and reaction structural parameters;

(b) it is possible the control of a system chaos degree by feedback interaction of nonlinear diffusion and sources (reaction) parameters.

\section{SOME APPLICATIONS OF LOCALIZED WAVES FOR MONITORING AND STABILITY ESTIMATION OF PRESSURE AND FLOW PULSES}

In paper (Djozjevitch, Sadov (1981)) basic principles of electrohaemodynamics (EHD) were developed and proposed system for determination of blood flow characteristics. EHD is mathematical modeling of the blood circulation combined with measurements of blood pressure and electrical conductance of a select part of the body. The main problem in the application of EHD to clinical measurements is how to obtain reliable values of all important cardiovascular parameters, such as diameters of arteries, modulus of elasticity of arterial walls, the length of blood vessels, etc.

EHD is capable of providing all the necessary parameters and, as a result, is used for measurements of cardiac output, cardiac index, stroke volume, systemic vascular resistance, useful power or work of the heart, etc. The technique is totally harmless; measurements are continuous and can be done for an indefinite period of time. EHD is further step in the development of electrical impedance phlethysmography. The common ground is the measurement of the variation of electrical impedance of a section of a human body as a function of time.

From analyze of the EHD principles we can conclude that this method may be combined with impulse response method, using the solitary waves exciting in the arteries. From our view, this approach may simplify the recognition process of the pressure and pulse propagation parameters and additionally allow to estimate the bounds of its stability.

In this section we consider a general mathematical model that may describe the cardiovascular interaction, determines pressure and flow (Westerhof, 1995).

It is suggested to use for description of the pressure and flow pulses in the region of low frequencies, and 
particularly of the solitary waves, a follows generalization of the Korteveg-de-Vries (KdV) equation:

$$
\frac{\partial u}{\partial t}+\sum_{i=1}^{3}\left[\frac{\partial}{\partial x_{i}} f(u)+\frac{\partial^{3}}{\partial x_{i}^{3}} g(u)\right]=0
$$

Here the solution $u(t, x)$ is to describe the vessel walls displacements, real functions $f(u), g(u)$ determine nonlinear structure of the blood flow and input impedance (resistance) of the vessels walls respectively. On case $f(u)=A u^{2}, g(u)=B u$ one obtains the traditional (week nonlinear) $\mathrm{KdV}$ equation. The structure of these functions should be improved by laboratory and clinical experiments. This model with nonlinear dispersion allow us to describe finite localized structures (so called compactons) or periodic switch waves that experimentally registered in large arteries by use of electro-impedance technique. It may be proven that on some conditions finite localized structures are highly dependent on blood viscosity and thus offer the potential for detection of clotting in blood vessels. This model is adaptable for elaboration of structure interaction control methods of the pulse propagation and patterns formation in arteries and vessels. By the special structure interaction of the nonlinear blood flow and input bio-impedance parameters may be constructed a new types of the solitary and switch waves. On the other hand, this model allow us to find the main relationships between structural parameters that determine the pulse propagation regularity and transition to deterministic chaos.

We assume that axisymmetric pressure pulse is moving with constant speed in the longitudinal direction of the tube. Then we may look fore solutions of this equation of the traveling-wave type:

$$
u=\varphi\left(\sum_{i=1}^{3} a_{i} x_{i}+v t\right)=\varphi(s)
$$

Here vis the constant velocity of the traveling wave, $a_{i}$ are the constants, that determines the wave propagation plane, $\sum_{i=1}^{3} a_{i}^{2}=1$. The desired function $\varphi(s)$ is governed by ordinary DE:

$$
v \varphi^{\prime}(s)+3\left[\frac{\mathrm{d}}{\mathrm{d} s} f[\varphi(s)]+\frac{d^{3}}{\mathrm{~d} s^{3}} g[\varphi(s)]=0\right.
$$

By integration on variable $s$ one may obtain the equation of reaction-diffusion type, that have investigated in previous chapters:

$$
\left.\frac{\mathrm{d}^{2}}{\mathrm{~d} s^{2}} g[\varphi(s)]+3 f[\varphi(s)]+v \varphi(s)\right\}-C=0
$$

here $C$ is the arbitrary parameter of integration, that shall to be determined from initial conditions.

Thus for this model all our results are applicable, which were considered in previous chapters. The finite localized solutions of type Eq. (24) may be used for description of the forward and backward waves in cardiovascular system [Westerhof, 1995]. Obtained exact analytic formulas in combination with the electro impedance monitoring allow to estimate the pulse velocity and other important parameters, that determine blood flow propagation. It is important, that monitoring in such a manner of the solitary waves may be used for quantitative estimation of the structural interaction of the vessel walls with the blood flow. By these estimations can be determined the bounds of the pressure and flow pulses stability and critical values of its parameters on that the pulse propagation can turns chaotic and breaks "through chaos to full annihilation" (see results of computational analyze in Figs. 7 and 8). The possibilities of applications of the solitary waves in cardiovascular monitoring were reported to International Conference in Washington (Kardashov and Einav, 1997).

The deplacements of the tube walls satisfy to the Korteweg-de-Vries equation (Hashizume, 1988; Demiray, 1997). On the other hand, according to Ohms law, electroimpedance of the flow and vessels cross-sectional area is proportional. This offers scope for application of the electro-impedance monitoring in association with solitary waves analyze for estimation of the vessels condition. By means of the electro-impedance technique we have registered the formation of periodic rheological graphics. We suggest the Eq. (25) and its exact solutions of Eqs. (27) and (28) as analytic models for description of experimentally obtained periodic transients. Taking into account that bio-impedance measurements are implemented by average process over measuring body area, from this result one can be reached the important conclusion on possibility of exciting of the coherent waves in the parts of the cardiovascular tree.

An amplitude and shape of the solitary wave are defined by elastic properties of the vessel walls. This give a possibility to determine by monitoring of solitary waves the important hemodynamic parameters such as diameters of arteries, modulus of elasticity of arterial walls, the length of blood vessels, etc.

\section{Acknowledgements}

The work is supported-part by Ministry of Absorption, The Drown Foundation and Herbert Berman Chair in Vascular Bioengineering. We thank Timur Kardashov for providing of computer simulation and graphs designing.

\section{References}

Biro, Z. (1997) "Attractors in a density-dependent diffusion-reaction model", Nonlinear Analysis, Theory, Meth. Applic. 29(5), 485-499.

Cohen, I., Golding, I., Kozlovsky, Y. and Ben-Jacob, E. (1998) "Continuous and discrete models of cooperation in complex bacterial colonies", Proc. of the 9th Intern. Symposium, Turkey.

Demiray, H. (1997) "Solitary waves in initially stressed thin elastic tubes”, Int. J. Nonlinear Mech. 32(6), 1165-1176.

Diaz, J. I. (1985) Nonlinear partial differential equations and free boundaries, Vol. 1 Elliptic Equations, Research Notes in Mathematics, (Pitman, New York) Vol. 106. 
Djordjevitch, L. and Sadove, M.S. (1981) "Basic principles of Electrohaemodynamics", J. Biomed. Engng. 3, 25-34.

Einav, S., Paz, D. and Elad, D. (1990) "Numerical simulation of blood flow through brunches", In: Liepsch, D., ed, Biofluid Mechanics: Blood Flow in Large Vessels (Springer, Berlin), pp. 501-511.

Elad, D., Katz, D., Kimmel, E. and Einav, S. (1991) "Numerical schemes for unsteady fluid flow through collapsible tubes", J. Biomed. Engng. $13,10-18$.

Gilmore, R. (1981) Catastrophe theory for scientists and engineering (Willey, New York) Vol. 1/2.

Gontar, V. (1993) New theoretical approach for physicochemical reactions dynamics with chaotic behavior Chaos in Chemistry and Biochemistry, (World Scientific, London), pp. 225-247.

Gontar, V. (1997) "Theoretical foundation for the discrete dynamics of physicochemical systems: chaos, self-organization, time and space in complex systems”, Discrete Dynam. Nat. Soc. Vol. 1, 31-43.

Gontar, V. (2000) "Entropy principle of extremality as a driving force in the discrete dynamics of complex and living systems", Chaos, Solitons Fractals 11, 231-236.

Gontar, V. and Ilin, I. (1991) "New mathematical model of physiochemical dynamics", Contr. Plasma Phys. 31(6), 681-690.

Hashizume, Y. (1988) "Nonlinear pressure wave propagation in arteries", J. Phys. Soc. Japan 57(12), 4160-4168.

Kardashov, V. (1993) "Generalized eigenvalue problems, stationary regimes and regimes with peakings of some strongly nonlinear nonstationary processes", Differen. Uravn. 29(3), 597-604.

Kardashov, V. (1999) "Finite control of nonlinear unsteady-state singular processes", Nonlinear Anal. 38, 361-374.

Kardashov, V. and Einav, Sh. (1997) "Solitary waves: applications to hemodynamics and cardiovascular monitoring", Int. J. Cardiovascular Med. Sci. 1(1).
Kolmogorov, A.N., Petrovskiy, G.I. and Piskunov, N.S. (1937) "Investigation of diffusion equation coupled with decreasing of matter quantity and application to biological problem", Bull. Mosc. Univ. A 1(6).

Li, Y.A. and Olver, J. (1997) "Convergence of solitary-wave solutions in a perturbed Bi-Hamiltonian dynamical systems. 1. compactons and peakons", Discrete Continuous Dynam. Syst. 3, 419-432.

Mikhailov, A.S. (1991) Foundation of synergetics, I. Distributed Active Systems (Springer, Berlin).

Phillips, R.J., et al., (1992) "Constitutive equation for concetrated suspensions that accounts for shear-induced particle migration", Phys. Fluids A 4(1), 30-40.

Rosenau, P. and Hyman, J. (1993) "Compactons: Solitons with finite wavelength”, Phys. Rever Lett. 70(5), 564-567.

Samarskiy, A.A., Galactionov, V.A., Kurdjumov, S.P. and Mikchailov, A.P. (1987) Regims with Peakings in Problems for Quasi-Linear Parabolic Equations (Nauka, Moscow).

Sanches-Garduno, F., Maini, Ph., K. and Kappos, Ph., E. (1996) “A Review on Travelling Wave Solutions of One-Dimensional ReactionDiffusion Equatioin with Non-Linear Diffusion Term", In: Maini, P.K., ed, FORMA Special Issue: Travelling Waves in Biology, Chemistry, Ecology and Medicine, Part 2 Vol. V.11,N1, pp. 1-80.

Talanov, V.I. (1983) Stimulated diffusion and cooperative effects in distributive kinetic systems Nonlinear Waves. Selforganization, (Nauka, Moscow).

Westerhof, N. (1995) "Cardiovascular interaction determines pressure and flow", Biological Flow (Plenum press, New York), pp. 227-253.

Zaslavskiy, G.M. and Sagdeev, R.Z. (1991) Week Chaos and QuasiRegular Structure (Nauka, Moscow), in Russia. 


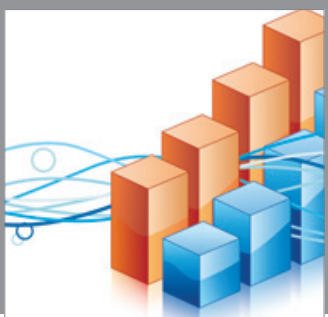

Advances in

Operations Research

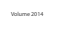

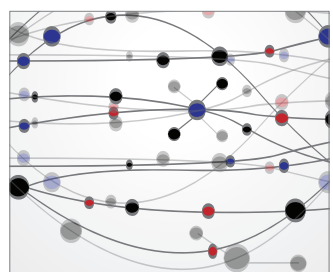

\section{The Scientific} World Journal
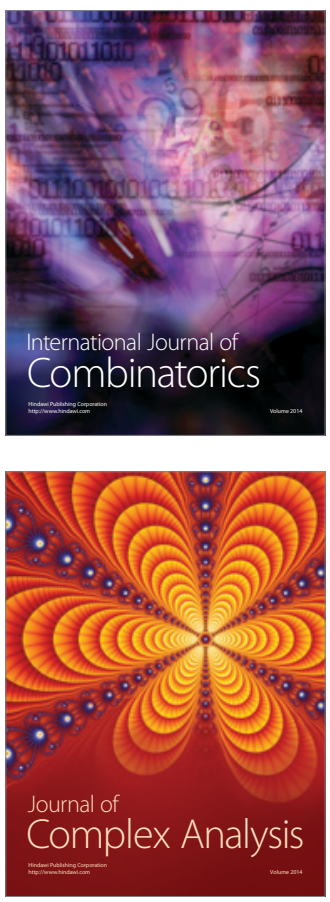

International Journal of

Mathematics and

Mathematical

Sciences
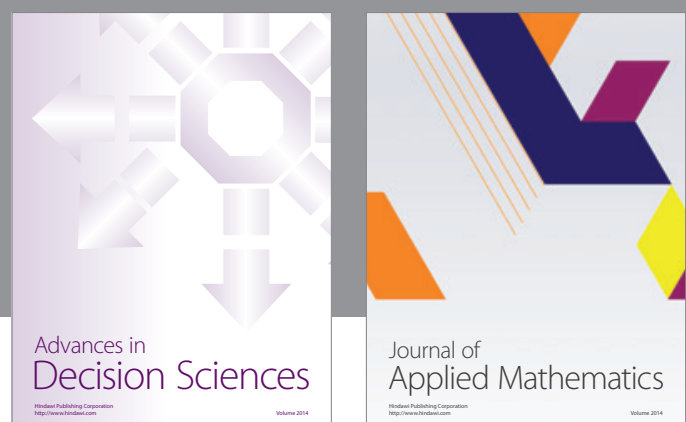

Journal of

Applied Mathematics
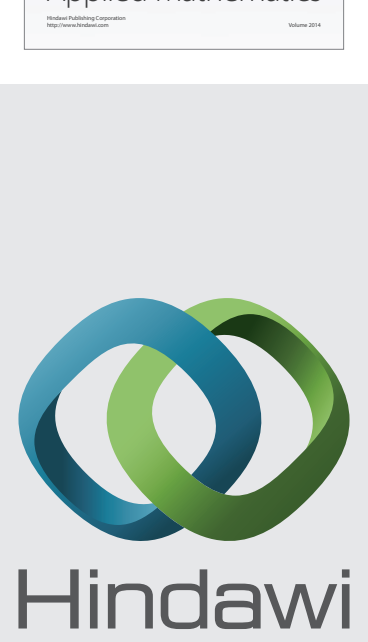

Submit your manuscripts at http://www.hindawi.com
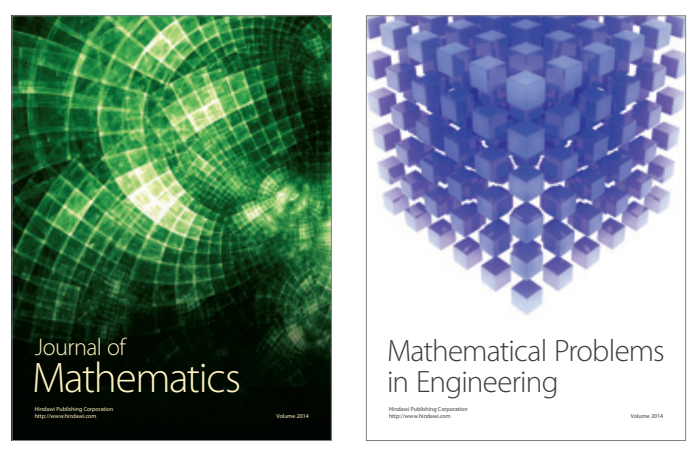

Mathematical Problems in Engineering
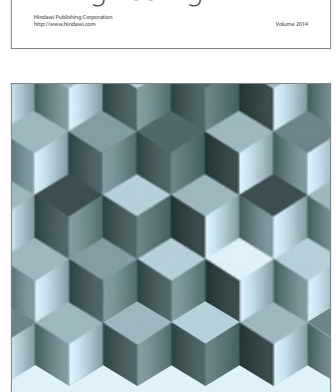

Journal of

Function Spaces
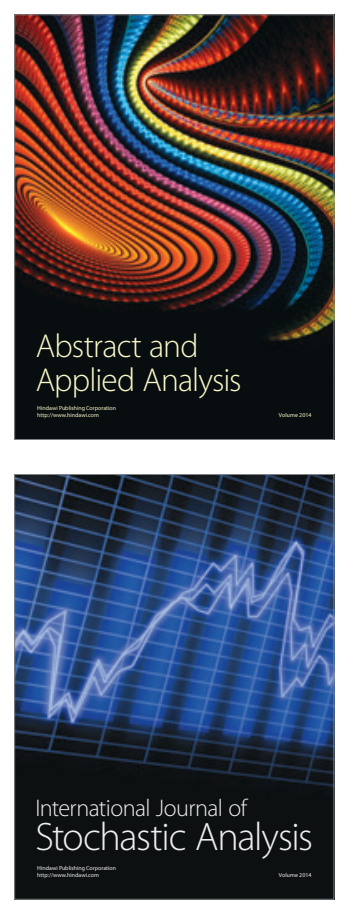

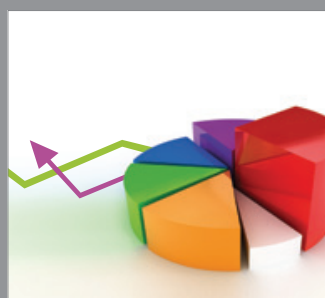

ournal of

Probability and Statistics

Promensencen
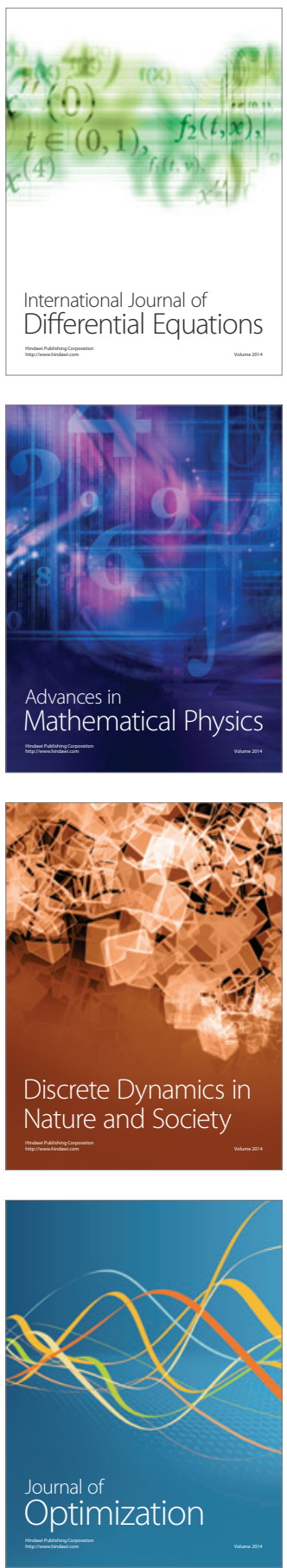